\title{
A PROCESS EVALUATION OF PATIENT CARE NEEDS USING THE POST-STROKE CHECKLIST: A PROSPECTIVE STUDY
}

Bhasker AMATYA, MD, MPH, DMedSc ${ }^{1-3}$, Alaeldin ELMALIK, MBBS, FAFRM (RACP) ${ }^{1,2}$, Su Yi LEE, PhD, MBBS, FAFRM $\left(\text { RACP) }{ }^{1-3} \text {, KRYSTAL SONG, PhD, MBBS, FAFRM (RACP) }\right)^{1-3}$, Mary P. GALEA, PhD, BAppSci (Physio), BA, Grad Dip Physio, Grad Dip Neurosci ${ }^{1-3}$ and Fary KHAN, MBBS, MD, FAFRM (RACP) ${ }^{1-3}$

From the ${ }^{1}$ Department of Rehabilitation Medicine, ${ }^{2}$ Department of Medicine and ${ }^{3}$ Australian Rehabilitation Research Centre, Royal Melbourne Hospital, Parkville, Victoria, Australia

Objective: To assess the utility of the modified Post-Stroke Checklist (mPSC) to identify impairments and care needs of patients with stroke (PwS) in an inpatient rehabilitation setting.

Methods: Prospective observational design with consecutive admission of PwS $(n=44)$ at a tertiary rehabilitation facility. The post-stroke checklist was administered at hospital discharge (T1) and 3 months post-discharge (T2). Furthermore, validated questionnaires assessed function and participation, including the Clinical Functioning Information Tool (ClinFIT) on admission (T0), T1 and T2.

Results: Participants' mean age was 67.7 years (standard deviation; SD) 14.6$), 58 \%$ of participants were female, and the mean length of inpatient stay was 32.7 days (SD 22.4). At T1, $80 \%$ and at T2 only $60 \%$ of participants reported $\geq 1$ strokerelated problem (mean 5.3 (SD 3.3) and 3.6 (SD 2.8), respectively). Half of participants were referred to physiotherapy/occupational therapy, and $36 \%$ to specialist clinics following discharge. The most prevalent problems included: life after stroke (62.2\%), fatigue (55.6\%), activities of daily living, and mobility (51.1\% each). Compared with T1, at T2 there was an observed reduction in all $\mathrm{mPSC}$ items, except pain and incontinence. Participants showed improved function at T1 and T2 (Extension Index, ClinfIT set), from TO to T1 and TO to T2 ( $p<0.001$, with large effect sizes).

Conclusion: The MPSC is feasible to implement in an inpatient rehabilitation setting and community. It can identify relevant stroke-related problems, and hence facilitate targeted intervention.

Key words: stroke; rehabilitation; care needs; Post-Stroke Checklist.

Accepted Dec 16, 2021; Epub ahead of print Jan 9, 2022

J Rehabil Med 2022; 54: jrm00259

Correspondence address: Bhasker Amatya, Department of Rehabilitation Medicine, Royal Melbourne Hospital, 34-54 Poplar Road, Parkville, Victoria 3052, Australia. E-mail: bhasker.amatya@mh.org.au

Stroke is a leading cause of death, and the single largest cause of long-term severe disability in Australia (1), with an estimated total lifetime cost burden of first-ever stroke of over Australian \$2 billion (2). The Global Burden of Diseases, Injuries, and Risk Factors

\section{LAY ABSTRACT}

Patients with stroke can develop significant long-term functional, communication and psychosocial disability, which can limit their daily activities and quality of life. Hence, regular follow-up is important to identify the patients' clinical needs after discharge from the hospital to the community. This study used a universal tool, the modified Post-Stroke Checklist (mPSC) to identify common persisting stroke-related issues of patients admitted to a rehabilitation unit to facilitate appropriate referrals during discharge. The MPSC is feasible to implement in an inpatient rehabilitation setting and community for treating clinicians to identify relevant strokerelated problems and provide appropriate intervention.

Study reports that stroke was the top-ranked cause of disability-adjusted life-years (DALYs) in adults age 50 years and over in 2019 (3). Despite improved survivorship following a stroke, patients with stroke $(\mathrm{PwS})$ can experience significant long-term functional, communication and psychosocial disability, which limits daily activity and participation. Over two-thirds of $\mathrm{PwS}$ discharged home from hospital have persistent symptoms, requiring assistance with activities of daily living (ADL) at 6 months post-stroke $(4,5)$. Stroke is complex, and requires interdisciplinary integrated management in hospital and community, with consideration of determinants of the health system; and the changing nature of both the patient and system $(1,5)$.

Health service provision is a priority for improving stroke care, with a need for wide-scale service reconfiguration to improve the delivery of post-acute stroke care. Regular patient follow-up is integral to identifying the clinical need (and unmet need) after discharge from the hospital to the community (6-10). $\mathrm{PwS}$ report challenges accessing the right services at the right time in the community, due to lack of services, inadequate referral processes and/or insufficient communication with healthcare providers, carer strain, etc. $(7,9)$. This may lead to discontinuation of prescribed care and adverse health effects. Furthermore, fragmented organizational coordination of systems after discharge from the hospital can lead to inefficient care and/or unnecessary delays, with harmful effects, both for patients and for the organizations involved (11, 
12). A survey by the US National Stroke Association reported that $40 \%$ of stroke survivors acknowledged a lack of information on rehabilitation and recovery (10). Despite the Australian Stroke Guideline recommendation of regular review of stroke survivors (1), the evaluation of longer-term care needs in the subacute phase of stroke management is often overlooked, and many patients do not receive rehabilitation review or necessary treatment $(13,14)$.

The Post-Stroke Checklist (PSC), developed by the Global Stroke Community Advisory Panel (2012), and endorsed by World Stroke Organisation (14) is a patient-centred pragmatic checklist to structure the stroke review process to assist health professionals in identifying common persisting sequelae following stroke, for facilitation of referrals for care (14). Previous studies (in UK and Singapore) demonstrate that the PSC is a realistic feasible approach to identify poststroke problems and facilitate referrals to appropriate support services $(11,14-16)$. Similar results from Italy support the web version of the PSC in communitydwelling stroke patients (11). A recent study, which evaluated data across 7 countries, demonstrated that the PSC provides a standardized measurement approach for identifying treatable, persisting problems for post-stroke care (17). Other studies confirm that timely discharge follow-up processes improve patient outcomes, reduce hospital readmissions and associated healthcare costs $(5,18)$.

This study examined the utility of the modified PSC (mPSC) for clinicians and patients to identify impairments and care needs of PwS in an inpatient rehabilitation setting. The feasibility and clinical usefulness of the PSC in addressing these needs were evaluated. It is envisaged that the findings will facilitate proper planning for therapeutic decision-making for improved outcomes within the stroke-care continuum.

\section{METHODS}

\section{Setting and subjects}

This prospective study was a part of a larger implementation trial (conducted between July 2019 and April 2021) (19) in a quality improvement programme at the medically supervised 40-bed inpatient Rehabilitation Unit, Royal Melbourne Hospital (RMH), a tertiary referral centre in Victoria, Australia. Currently, RMH has well-established interdisciplinary rehabilitation programmes for stroke. This study was approved by the Institutional Ethics Committee (Human Research Ethics Committee (HREC) number 2019.119), in accordance with the Declaration of Helsinki and Strengthening the Reporting of Observational Studies in Epidemiology (STROBE) criteria for observational studies (20).

All PwS consecutively admitted to the RMH rehabilitation unit who fulfilled the inclusion criteria: confirmed diagnosis of first episode of stroke, $>18$ years of age, and ability and wil- lingness to give informed consent, were eligible to participate in the study. Those with severe cognitive issues or substantial neurological or psychiatric disorders that prevented informed consent were excluded. All eligible patients were invited to participate in this study by an independent nurse/researcher, who explained the purpose, benefits and risks of participation. Sufficient time was allowed for discussion of any questions raised by the participant. Participants were able to withdraw from the study at any time, but were not re-enrolled.

\section{Procedure}

The clinicians in the unit were trained in cognitive and functional ability assessments and received further formal training from the authors (AE, SL, FK), comprising an introduction to the $\mathrm{mPSC}$ and the study protocol. Consistent with routine clinical practice, every patient was assessed at admission (T0) by the interdisciplinary team and received an individualized rehabilitation programme based on clinical need. Further assessments were conducted at discharge from the ward (T1) and at 3-month post-discharge (T2) (telephone follow-up) using standardized instruments. These assessments took approximately 15-20 min. The staff did not prompt patients, but assisted those with difficulty in expressing their needs. The study comprised the following phases:

- Pre-implementation medical record audit. A retrospective discharge summary audit of randomly selected rehabilitation inpatients $(n=20)$ discharged from the ward, prior to patient recruitment for the study (February-June 2019). The information included: stroke-related problems documented during inpatient care, referrals and services listed at the time of discharge. This information identified various health concepts discussed between staff and patients at the time of discharge.

- Initial assessment (at admission) (TO). Within $24 \mathrm{~h}$ of admission to the service, routinely clinical information included: sociodemographic and stroke-related information (diagnosis, spasticity, pain, etc.), medications and co-morbidities. Furthermore, routinely used, validated outcome measures assessed patient function and dependency. These included: Functional Independence Measure (FIM), International Classification of Functioning, Disability and Health (ICF), Clinical Functioning Information Tool (ClinFIT) and EuroQuality of life (EQ-5D-5L).

- At discharge (T1). In addition to the outcome tools used at T0, the mPSC was used to assess patients' care needs and unmet needs (see measures below). This information was included in a comprehensive medical discharge summary, with the follow-up care plan.

- At 3-months follow-up (T2). The same measures as T0 and T1 were utilized. The PSC was administered again by a designated, trained researcher on the ward via telephone call to the patient. Furthermore, participant societal reintegration was assessed using the Community Integration Measure (CIM).

- Evaluation of the mPSC administration. A post-implementation evaluation assessed the clinicians' experience and satisfaction with the mPSC implementation. All rehabilitation physicians involved $(n=9)$ completed a structured satisfaction questionnaire, which included 6 items on: practicality; usefulness in identifying patients' needs; completeness of the content; enhancement of communication; helpful in decision-making and referrals; and overall satisfaction. The clinicians rated each item on a 0 (strongly disagree) to 10 (strongly agree) numerical rating scale. 


\section{Measures}

The outcome measures used were as follows:

Modified Post-Stroke Checklist. The mPSC (Canadian version) (21) includes 13 post-stroke problem areas: secondary prevention, activities of daily living (ADL), mobility, spasticity, pain, incontinence, communication, mood, cognition, life after stroke, personal relationships, fatigue, and other challenges. Each item comprises a dichotomous response scale: "Yes" (requiring follow-up with the appropriate action) and "No" (requiring review at next assessment). At discharge (T1) the assessors evaluated participants "since their stroke" for each mPSC item, while at 3-month follow-up (T2) assessment participants were assessed "since the last assessment" (Appendix S1).

Functional Independence Measure. The FIM (22) (18 categories): motor domain (13 items) assesses the level of function in 4 subscales: Self-care, Transfers, Locomotion, and Sphincter control; and cognition domain (5 items) in 3 subscales: communication, psycho-social and cognition. Participants are rated on each item on a scale of 1 to $7(1=$ total assistance, $4=$ requires physical assistance, $5=$ needs supervision, $6=$ modified independence, $7=$ independent) by trained staff. The score reflects dependency in each area measured (22).

ICF Clinical Functioning Information Tool (ClinFIT) comprises 30 categories that define a minimum set of information on "functioning" and "disability" collected across health conditions along the continuum of care (23): 9 items in the "Body Functions" domain: and 21 items in the "Activities and Participation" domain (24-26). These categories, accompanied by clinically meaningful descriptions, were used by clinicians to assess patient functioning on an 11-point numerical rating scale $(0=$ no problem to $10=$ complete problem $)$. The ClinFIT set demonstrated good internal consistency reliability, with a Cronbach alpha coefficient of 0.97 (Appendix S2).

Euro-Quality of Life. The EQ-5D-5L (27) has 5 health dimensions: mobility, self-care, daily activity, pain/discomfort, and anxiety/depression, and a sixth item of a visual analogue scale (VAS) for current overall health. Responses for these 5 dimensions are divided into 5 ordinal levels: no problems, slight problems, moderate problems, severe problems, and extreme problems. Furthermore, participants rated their current overall health in a sixth item, a VAS on a $20-\mathrm{cm}$ vertical scale from 0 (the worst health state they can imagine) to 100 (the best health state on that day they can imagine). The EQ-5D index score was generated using 5 level-responses to each item based on a published crosswalk algorithm, which provides index-based scores ranging from -0.594 to 1.0 (in the UK population), with lower values signifying worse health (27).

Community Integration Measure. The CIM (28) has 10 declarative statements to assess perceived community integration in 4 domains: general assimilation, supports, occupation, and independent living. Respondents rated each statement on a Likert scale ( $1=$ always disagree, $5=$ always agree $)$ to give a total score out of 50. Higher scores indicated greater community integration.

\section{Statistical methods}

A preliminary analysis assessed data distribution using the Shapiro-Wilk test. De-identified information was coded, entered into a database and descriptive statistics were reported. Frequency of development of new disorders, e.g. functional deterioration, new symptoms and factors contributing to changes, were identified. Cross-sectional sample profiling was conducted initially through descriptive analysis, and percentages for different answers provided by participants were computed. A qualitative analysis was used for the discharge summary audit and satisfaction survey responses; and interpreted descriptively.

Since data were not normally distributed for the outcome measures, a series of non-parametric tests (Wilcoxon signed-rank test) determined the differences between T0-T1 and T0-T2. Effect size statistics ( $\mathrm{r}$ ) were calculated by dividing the $\mathrm{z}$ score by the square root of $\mathrm{N}$ (total number of cases) and assessed against Cohen's criteria $(0.1=$ small, $0.3=$ moderate, $0.5=$ large effect) (29). To detect changes in ClinFIT scores between admission (T0) and different assessment time-points ( $\mathrm{T} 1$ and $\mathrm{T} 2$ ), the count-based "Extension Index" (EI) was calculated for the total ClinFIT set, and its "body function (b)" and "activity and participation (d)" domains. The EI is calculated as [(the count of categories with qualifier $1-10 /$ the number of entire categories) $\times 100$ ] (30). The transformed value ranged from 0 to 100 , with the lowest value representing no issues with body function, and no limitation/restriction in activity and participation $(30,31)$. The Wilcoxon signed-rank test compared the EI of the ClinFIT set between T0-T1 and T0-T2 periods. A $p$-value $<0.05$ was considered statistically significant. All analyses used the IBM SPSS Statistics Package Version 27 (Chicago, IL, USA).

\section{RESULTS}

A total of 44 eligible $\mathrm{PwS}$ consecutively admitted after 7-14 days after the first ever stroke to the rehabilitation ward, who met the inclusion criteria and provided written consent were recruited. During the study, 2 participants were uncontactable at the 3-month followup (T2) assessment.

\section{Sociodemographic and clinical characteristics}

Descriptive statistics for patient demographic and clinical characteristics are in Table I. The participants' mean age was 67.7 years (SD 14.6) (range 23.8 to 92.1 years), predominantly female $(n=26,58 \%)$, and Caucasian $(n=37,84 \%)$. Only 8 participants were employed at T0. Most had had an ischaemic stroke $(n=38$, $84 \%)$ and $(96 \%)$ reported 1 or more comorbidities, with hypertension $(n=38,84 \%)$, followed by depression $(n=8,18 \%)$ and diabetes $(n=5,11 \%)$ being the most common. More than half of the participants $(n=26$, $59 \%$ ) were taking $>3$ medications) at T0. The common impairments reported were: upper and/or lower limb weakness, ( $82 \%$ for both), cognitive impairment $(n=27,61 \%)$, sensory $(n=19,43 \%)$, speech $(n=16$, $36 \%)$, and vision issues $(n=24,52 \%)$. The majority had fatigue $(n=39,89 \%)$, pain $(n=30,68 \%)$, and were at risk of falling $(n=41,93 \%)$ (Table I).

\section{Retrospective audit of discharge summaries}

The majority of concepts documented in the discharge summaries were directly or partially linked with the mPSC items reported by patients' at T1 (Table II). 
Table I. Characteristics of participants $(n=44)$

\begin{tabular}{|c|c|}
\hline \multicolumn{2}{|l|}{ Characteristics } \\
\hline Age, years, mean (SD) [range] & $67.7(14.6)[23.8-92.1]$ \\
\hline Female, $n(\%)$ & $26(57.8)$ \\
\hline Ethnicity - Caucasian, $n(\%)$ & $37(84.1)$ \\
\hline NESB, $n(\%)$ & $9(22.0)$ \\
\hline Married/partner, $n(\%)$ & $24(53.3)$ \\
\hline \multicolumn{2}{|l|}{ Living with, $n(\%)$} \\
\hline Alone & $11(24.4)$ \\
\hline Partner/family & $33(73.3)$ \\
\hline \multicolumn{2}{|l|}{ Education, $n(\%)$} \\
\hline Secondary & $25(55.6)$ \\
\hline Tertiary & $9(20.0)$ \\
\hline Employed, $n(\%)$ & $8(17.8)$ \\
\hline Carer, n (\%) & $2(4.5)$ \\
\hline LOS, days, mean (SD) & $32.7(22.4)$ \\
\hline \multicolumn{2}{|l|}{ Stroke type, $n(\%)$} \\
\hline Ischaemic & $28(63.6)$ \\
\hline Haemorrhage & $16(36.4)$ \\
\hline Polypharmacy (on $\geq 3$ medication), $n(\%)$ & $26(59.1)$ \\
\hline \multicolumn{2}{|l|}{ Co-morbidities, $n(\%)$} \\
\hline Hypertension & $38(84.4)$ \\
\hline Diabetes & $5(11.1)$ \\
\hline Depression & $8(17.8)$ \\
\hline \multicolumn{2}{|l|}{ Impairments/symptoms, $n(\%)$} \\
\hline Spasticity & $4(9.1)$ \\
\hline Hemiparesis & $34(77.3)$ \\
\hline Upper limb impairment & $37(84.1)$ \\
\hline Lower limb impairment & $37(84.1)$ \\
\hline Cognition & $27(61.4)$ \\
\hline Sensory & $19(43.1)$ \\
\hline Speech & $16(36.4)$ \\
\hline Hearing & $9(20.5)$ \\
\hline Visual & $23(52.3)$ \\
\hline Falls risk & $41(93.2)$ \\
\hline Pain & $30(68.2)$ \\
\hline Fatigue & $39(88.6)$ \\
\hline Bladder issues & $5(11.4)$ \\
\hline Bowel issues & $7(15.9)$ \\
\hline
\end{tabular}

LOS: length of stay; NESB: non-english speaking background; SD: standard deviation.

The range of post-stroke issues documented in the discharge summaries: $100 \%$ had difficulty with ADL (basic and community), transfer $(n=17,85 \%)$ and mobility problems $(n=16,80 \%)$, fatigue $(n=15,75 \%)$, cognitive dysfunction $(n=15,75 \%), \operatorname{mood}(n=13$, $65 \%)$ and walking $(n=12,60 \%)$.
Table II. Summary of frequency of stroke-related problems documents in discharge summary $(n=20)$

\begin{tabular}{|c|c|c|}
\hline Post-stroke issues & $n$ & $\%$ \\
\hline$A D L^{a}$ & 20 & 100 \\
\hline Transfer $^{a}$ & 17 & 85 \\
\hline$C A D L^{a}$ & 17 & 85 \\
\hline Mobility ${ }^{a}$ & 16 & 80 \\
\hline Fatigue $^{a}$ & 15 & 75 \\
\hline Cognitive impairments ${ }^{a}$ & 15 & 75 \\
\hline Mood $^{a}$ & 13 & 65 \\
\hline Walking ${ }^{a}$ & 12 & 60 \\
\hline Sleeping problems & 9 & 45 \\
\hline Bowel impairments $^{a}$ & 9 & 45 \\
\hline Communication $^{a}$ & 9 & 45 \\
\hline Transport & 8 & 40 \\
\hline Driving & 8 & 40 \\
\hline Balance $^{\mathrm{b}}$ & 7 & 35 \\
\hline Bladder $^{a}$ & 7 & 35 \\
\hline Swallowing difficulties ${ }^{b}$ & 7 & 35 \\
\hline Social activity ${ }^{a}$ & 6 & 30 \\
\hline Malnutrition & 6 & 30 \\
\hline Pain $^{\mathrm{a}}$ & 5 & 25 \\
\hline Caring for others ${ }^{b}$ & 3 & 15 \\
\hline Employment $^{\mathrm{a}}$ & 3 & 15 \\
\hline Visual impairments & 3 & 15 \\
\hline Hearing impairments & 1 & 5 \\
\hline Others & 2 & 10 \\
\hline
\end{tabular}

${ }^{a}$ Concept directly measured by the modified Post-Stroke Checklist (mPSC). ${ }^{\mathrm{b}}$ Concept partially measured by the MPSC.

ADL: activities of daily living; CADL: community activities of daily living.

\section{Stroke-related problems}

All participants reported receiving medical advice regarding secondary stroke prevention, medications and health-related lifestyle changes (mPSC 1) at T1, while at T2 4 participants $(9 \%)$ reported not receiving further information (Fig. 1). One or more post-stroke health problems were reported by $80 \%$ of participants at discharge (T1). These post-stroke issues at T1 tended to decrease at 3-month follow-up (T2), and were reported by only 26 participants $(60.1 \%)$. Compared with $\mathrm{T} 1$, at $\mathrm{T} 2$ this reduction was observed in all $\mathrm{mPSC}$ items except pain (mPSC 5) and incontinence (mPSC

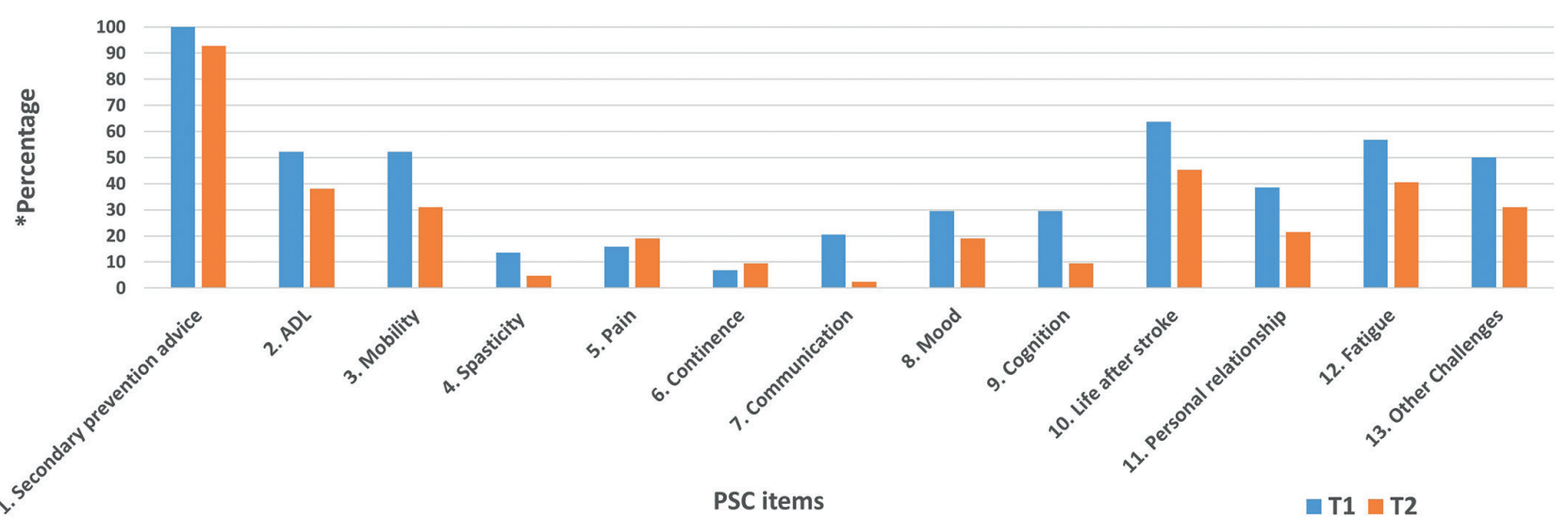

Fig. 1. Prevalence of stroke-related problems identified with the modified Post-Stroke Checklist (mPSC) items at discharge (T1) and 3-month follow up (T2). *A number expressed as a percentage who responded "yes" to each PSC item. ADL: activities of daily living; T1: Discharge; T2: 3-month follow-up. 
6) treatment (Fig. 1). The mean number of post-stroke problems reported by participants across the mPSC items at $\mathrm{T} 1$ was $5.3 \pm 3.3$ (range $1-12$ ) and at 3 months after discharge $3.6 \pm 2.8$ (range $0-11$ ). At T1, the most prevalent reported problem since stroke was "life after stroke" (62.2\%), followed by "fatigue" (55.6\%), "ADL" (51.1\%) and "mobility" (51.1\%).

At discharge (T1), as per routine practice, all discharge summary letters were sent to treating general practitioners (GP) in the community. Of these, 6 participants were referred for quick medical review within 2 weeks. Most participants $(n=33,75 \%)$ were referred to their local community-based rehabilitation programmes. More than half $(n=23,52 \%)$ were referred to physiotherapists (PT), followed by occupational therapists (OT) $(n=21,48 \%)$, stroke and other specialist clinics ( $\mathrm{n}=16,36 \%$ for both) (Fig. 2).

At 3-month follow-up (T2), most participants $(n=39,93 \%)$ had had a medical review by their GPs or other medical professionals. The most common rehabilitation interventions involved PT $(n=35,83 \%)$, followed by OT ( $n=27,64 \%)$, speech therapy $(n=8$, $19 \%)$, social worker $(n=7,17 \%)$ and dietician $(n=6$, $14 \%)$. Six participants also reported doing regular home exercise, walking or gardening. Two participants reported ongoing spasticity and were awaiting botulinum-toxin injections.

\section{Outcome measurement change scores overtime}

Summary data for all outcome measures at different assessment points are provided in Table II. Participants' median (Md) length of stay at the rehabilitation unit was 71 days (interquartile range (IQR): 15, 48 days). As expected, at discharge (T1), there was a significant improvement in functional and cognitive outcomes, as confirmed by FIM motor total and all subscales: "self-care", "sphincter", "locomotion", "mobility" ( $p<0.001$ for all), with medium to large

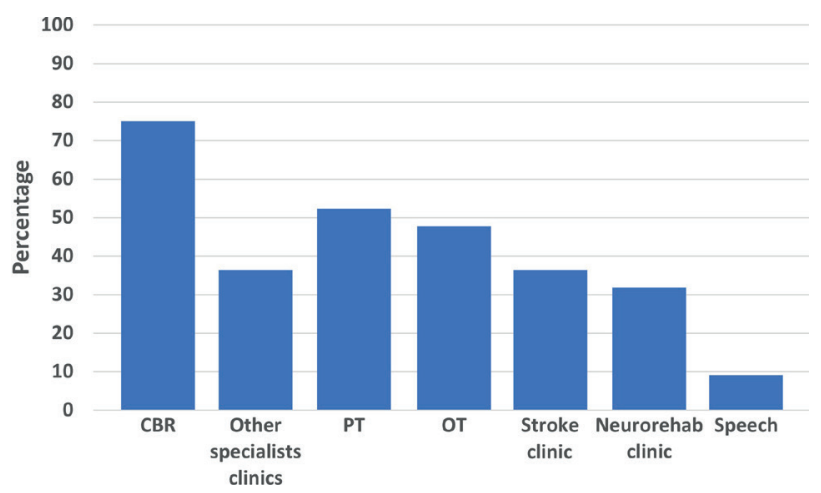

Fig. 2. Frequency of participants referred to various specialists at discharge (T1) based on their needs $(n=44)$. CBR: community-based rehabilitation; OT: occupational therapists; PT: physiotherapists. effect size $(\mathrm{r}=0.49-0.60)$; and FIM cognition "total", and "communication", "psychosocial" and "cognition" subscales $(p<0.001)$ with moderate to medium to large effect size $(\mathrm{r}=0.46-0.52)$. The quality of life $(\mathrm{QoL})$ and overall health of participants improved significantly (EQ-5D and overall health scores) $(p<0.001$ for all), with large effect sizes $(r=0.53-0.61)$.

Forty participants (91\%) were discharged home, and most maintained their functional and cognitive improvement (FIM scores) at 3 months follow-up (T2). Significant functional improvements were maintained by most participants, as measured by FIM motor "total" and each subscale ( $p<0.001$ for all) with medium to large effect size $(r=0.42-0.59)$, and for the FIM cognition "total" and each cognition subscales ( $p<0.001$ for all), the magnitude of effect improved to large $(\mathrm{r}=0.50-0.57)$. The improvement in QoL and overall health of participants remained statistically significant $(p<0.001$ for all); however, the magnitude of effect decreased from moderate to large effect size $(\mathrm{r}=0.46-0.57)$ (Table II). Furthermore, participants reported adjustment to community living after a stroke at T2 [CIM total (Md (IQR): $46.5(40,50)$ ].

Most participants reported at least 1 issue related to ClinFIT categories at admission (T0). The most common ClinFIT categories (reported by $>95 \%$ participants) included: "Emotional functions" (b152), "Carrying out daily routine" (d230), "Walking" (d450), "Moving around" (d455), "Assisting others" (d660), "Recreation and Leisure" (d920). Most participants showed improvement from admission to both assessment time-points in their body function, (b) and activity and participation (d) domains (Fig. 3). There was a significant improvement in participants in ClinFIT raw total score at both $\mathrm{T} 1$ and $\mathrm{T} 2$ assessment points $(p<0.001$ for both) with large effect sizes $(\mathrm{r}=0.62$ and 0.71 , respectively). There were significant changes in the Extension Index (EI) in the entire ClinFIT set from admission (T0) to discharge (T1) $(\mathrm{z}=-4.83, p<0.001)$ and from $\mathrm{T} 0$ to $\mathrm{T} 2(\mathrm{z}=-5.37$, $p<0.001)$, with large effect sizes $(0.52$ and 0.79 , respectively). The changes in the EI were significant for both ClinFIT "b" and "d" domains at both T1 and $\mathrm{T} 2$, indicating that participants had fewer issues with their body function and less restriction in everyday activities (Table III).

\section{Satisfaction with the mPSC implementation}

The majority of treating clinicians $(87 \%)$ involved in the study were satisfied using mPSC (score $>8$ ). The mPSC was feasible to use in busy clinical settings and assisted in identifying patient needs, improved communication, therapeutic decision-making and 


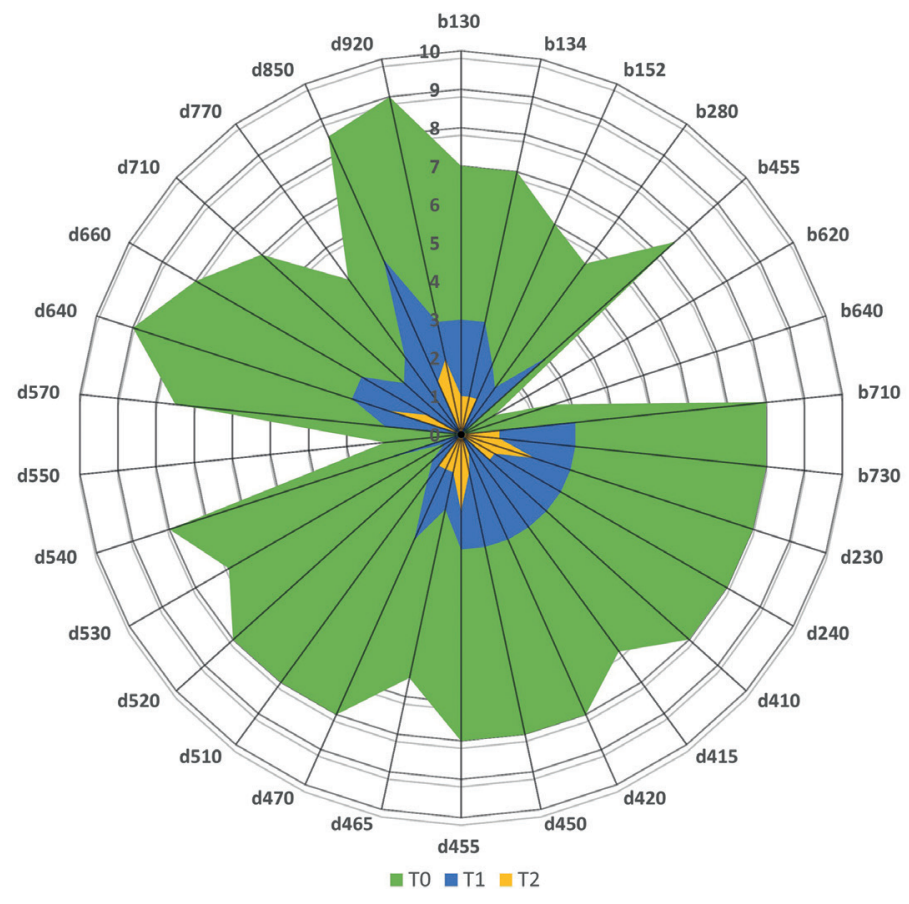

Fig. 3. Composite Radar Chart illustrating the median scores for each Clinical Functioning Information Tool (ClinFIT) item at admission (T0), discharge (T1) and 3 months (T2). The composite radar chart provides a graphic representation of the functional profile from the ClinFIT data. The 30-scale items are arranged as spokes of a wheel (codes out the circumference), with International Classification of Functioning, Disability and Health (ICF) qualifiers from $0=$ no problem to $10=$ complete problem, running from the centre outwards. Green-shaded area represents the median admission (T0) scores $(n=44)$, blue-shaded area the median discharge (T1) scores $(n=44)$ and yellow-shaded area the median scores at 3-month follow-up (T2) $(n=42)$. b130-b730 represent 9 ClinFIT "Body function" categories and d230-d920 represents 21 ClinFIT "activity and participation" categories. referrals for the patient. However, $53 \%$ of clinicians indicated (scores $\geq 7$ ), suggesting that the mPSC may have missed a few important stroke-related problems (Fig. 4).

\section{DISCUSSION}

Regular assessment of clinical outcomes in routine clinical practice is integral for good practice and de-

Table III. Change scores in subscales for measurement scales overtime

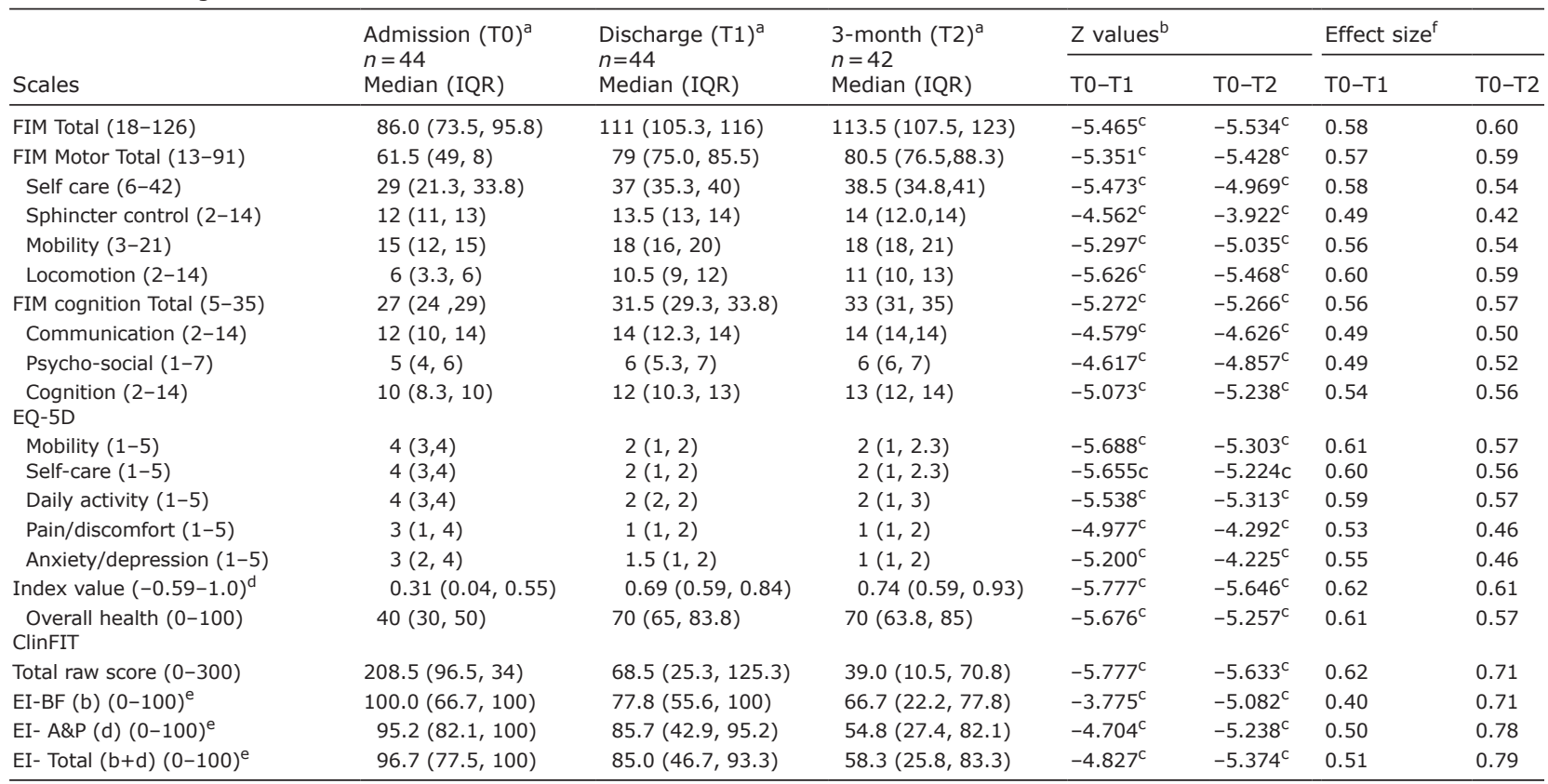

${ }^{a}$ All values are provided as median (interquartile range; IQR). ${ }^{b}$ Wilcoxon signed-rank test. ${ }^{c}$ Correlation significant at all levels $<0.001$ level $\left(2\right.$-tailed). ${ }^{d}$ EQ-5D index-based summary score (UK). ${ }^{e}$ Extension index was calculated as: (number of problem categories/entire number of categories) $\times 100$. ${ }^{f}$ Effect size statistics (r) Cohen's criteria: $0.1=$ small, $0.3=$ medium, $0.5=$ large effect.

A\&P: Activity and participation; BF: Body function; ClinFIT: Clinical Functioning Information Tool; EQ-5D: Euro-Quality of Life scale; FIM: Functional Independence Measure; $n$ : total number. 


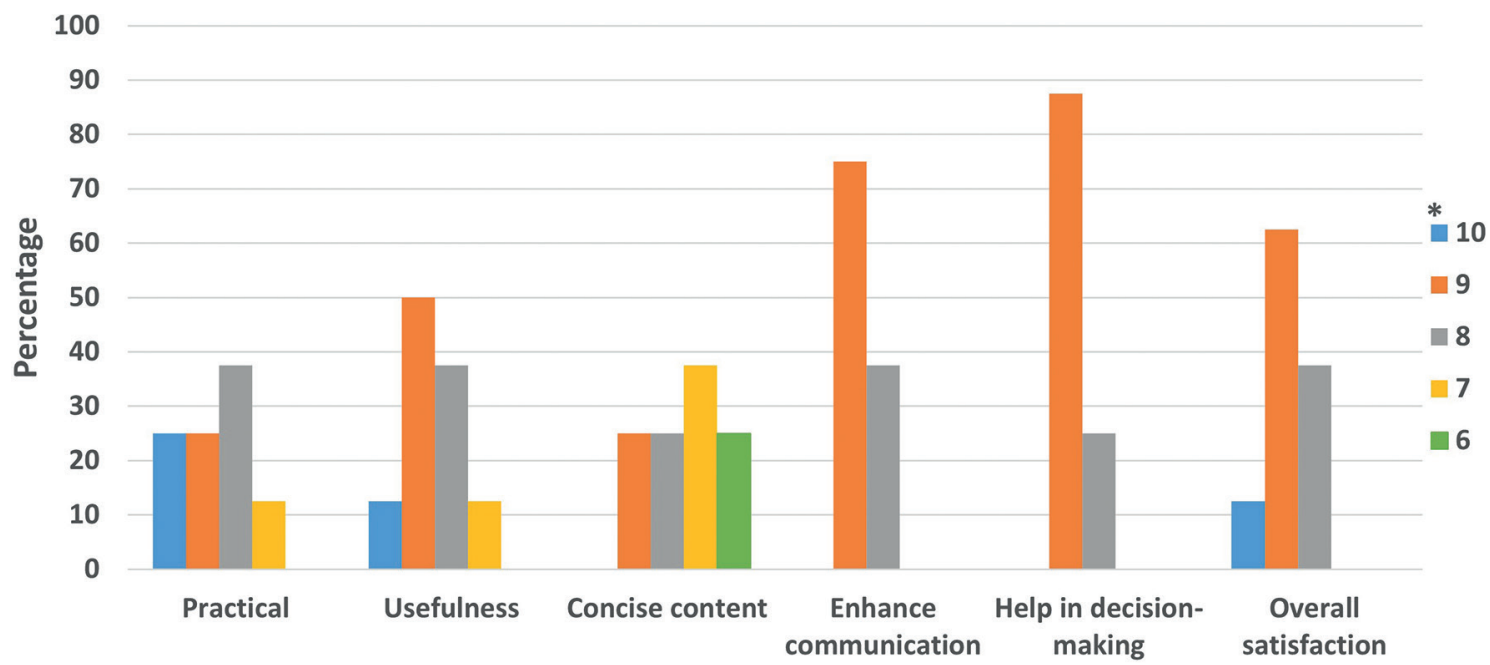

Fig. 4. Clinicians' view of the modified Post-Stroke Checklist (mPSC) $(n=9)$. *Numerical rating scale: 0 (strongly disagree) to 10 (strongly agree). There was no response for ratings $0-6$, hence they are excluded from the figure.

livery of quality healthcare. This prospective study demonstrated the feasibility and clinical usefulness of the mPSC in the systematic evaluation of health outcomes of first stroke survivors in a busy public hospital rehabilitation setting, in the Australian context. After completion of the inpatient rehabilitation programme, there was a significant functional improvement, psychosocial adjustment and enhanced QoL (as measured by FIM, EQol-5D-5L) (with large effect sizes); however, many participants reported persisting stroke-related issues at the time of discharge and at 3-month follow-up. The mPSC facilitated the recording of information in a systematic structured manner, and enabled streamlined referrals and treatment for these post-stroke problems. There was a marked reduction in the mean problems reported by participants across the mPSC items at the 3-month follow-up after discharge. The treating clinicians were satisfied with the MPSC, reported improved communication with patients and timely referrals to appropriate clinical services.

The study used a multi-pronged approach to assess the patient disease trajectory including retrospective audits, prospective assessments and clinician satisfaction survey. The pre-implementation retrospective audit showed that the majority of clinical concepts documented in the discharge summaries were directly or partially linked to the mPSC items. The World Health Organization (WHO) ICF ClinFIT set has exhaustive categories, in the "body function" and "activity and participation" domains (32), which describe patients' functional status comprehensively in inpatient rehabilitation settings and across the continuum of care (33). Furthermore, it was sensitive to change over time, generating an interval score (EI) (from admission to discharge and 3-month post-discharge), which provided information on various aspects of function and disability. Most participants (over 95\%) reported various issues specifically in the "activity and participation" components of the ClinFIT (cognition, ADL, walking, recreation and leisure, etc.). Importantly, this data was consistent with information from the PSC.

The PSC is globally endorsed by various stroke networks (and the WHO) to support improved management of PwS $(11,17,34,35)$, and is validated, with several studies supporting its feasibility and practicality in different settings $(11,14,16,17,34-38)$. One cross-sectional study (from 7 different countries) used the PSC to compare post-stroke sequelae (17), and listed these as issues relating to: cognition, life after stroke, mood, mobility and ADL (17). These results are consistent with the findings of the current study. Ullberg et al. (38), in a prospective follow-up study ( $n=200)$ mapping stroke-related issues using PSC in a Swedish cohort, reported that prevalence of stroke-related problems ranged from low ("spasticity" $7.3 \%$ ) to high ("secondary prevention" $42.2 \%$, "mood" $38.2 \%$, "cognition" $37 \%$, "fatigue" $48.5 \%$, "life after stroke" $44.5 \%$ ) at 3 months post-discharge. Another recent study $(n=197)$ reported that "mood", "pain" and "cognition" were the most prevalent stroke-related problems, followed by "life after stroke", "spasticity" and "ADL" at 3 months post-discharge (34). The prevalence of deterioration of "ADL", "mobility", and "communication" increased more than 3 -fold at 6 months (34). Similar results were reported in an Italian community-dwelling cohort (11). Many of these results are consistent with our findings using the mPSC. Some values, (such as the prevalence of spasticity and secondary prevention advice) were different from the previous reports, probably due to 
differences in the use of the PSC version, healthcare and welfare systems, and culture. Furthermore, the current study cohort comprised patients admitted to rehabilitation with their first stroke after acute treatments (7-10 days). This was different from the study cohort assessed in a cross-sectional study comparing PSC data across all 7 different countries (17) and other studies with longer intervals since acute events $(10$, $14,16)$. In contrast to the aforementioned studies, this study evaluated key patient concerns using mPSC at the time of discharge to streamline referrals and care interventions in the community, with a 3-month followup. The cohort reported ongoing fatigue and mobility issues at both discharge and 3-month follow-up, which probably contributed to persisting worse participation (life after stroke). This shows the inter-relationship of many of the mPSC items, and signifies the need for their interpretation in conjunction with each other. The prevalence of patient-reported problems decreased in the current study cohort at 3 months, which might be due to patient adherence to referrals and engagement in suggested interventions. This also might be due to better clinician-patient communication resulting in better patient understating of their issues. Moreover, despite the fact that more than half (52\%) were referred to PT at discharge (T1), the majority (83\%) reported that they were involved in some form of physical activity. Furthermore, as post-discharge follow-up was a short period (3 months) by telephone, persisting stroke-related problems at 3 months cannot be ruled out despite improvements being gained. It was beyond the scope of this study to follow the participants beyond 3 months post-discharge.

Pain and continence are common clinical problems and are often undertreated $(39,40)$. Several related factors include: older age, female sex, motor weakness, cognitive impairments, etc. $(39,40)$. Despite the reduction in other stroke-related issues in the current study cohort, pain (mPSC 5) and incontinence (mPSC 6) issues were persistent at 3-month followup. The current study cohort was largely female and older (mean age 67.7 years (SD 14.6)), with one-half predominantly reporting mobility and fatigue issues. The mPSC identified these problems at T1, and these patients were receiving ongoing care at T2. Post-stroke pain and incontinence are multifactorial, complex, and need comprehensive management (pharmacological and non-pharmacological) over time.

The needs of PwS are often not reviewed comprehensively, and without the required care services (11, 12). This pragmatic clinical observational study was part of a continuous practice improvement process to improve care, education and patient self-management. Although the rehabilitation service routinely reinforces patient adherence by a post-discharge phone call pro- cess (commencing 2 weeks after discharge from the service), this process is ad hoc, based on the available clinical information and not always documented in a structured manner (personal communication with clinicians). Therefore, implementation of the structured mPSC checklist provided consistency and improved clinical documentation for timely referrals and care delivery. It also enabled the identification of patient factors associated with adherence to the post-discharge management in the community and/or gaps in service provision in the stroke care system at large.

This study has a number of potential limitations. First, this is a longitudinal observational study without a control group, which limits the ability to draw causal relationships between the care process and improvements in patient outcomes. However, various other functional and quality of life measures were used, which showed significant improvement in patient outcomes at discharge, and were maintained at 3 months post-discharge. Secondly, participants were a small selective cohort admitted to a single tertiary institution, which may limit the generalizability of findings. However, our rehabilitation unit receives patients from a wide geographical population in Victoria, Australia. Furthermore, participants in this study were complex in terms of disease severity, symptoms and comorbidities, reflecting the true picture of the sub-acute stroke patient population. This study was conducted amidst the COVID-19 pandemic with multiple lockdowns and restrictions (such as travel restrictions and stay at home orders, workplace and business closures, etc.). This impacted participant recruitments, attrition and compliance to the prescribed interventions. Many community services ceased during lockdown, and used online services. Despite comprehensive assessment using the PSC (and other measures), some patients may not have received adequate and/or timely referrals. Thirdly, the mPSC version (Canadian) used in this study is yet to be validated according to the standardized procedure performed when developing the original PSC. This might have implications when interpreting the results. However, the mPSC was modified for local context and contains all items from the original PSC with 2 additional items. Fourthly, although the validated measures used are broad, some important concepts may have been missed, which are not included in our assessment battery. All measurement scales assessed the current situation to reduce recall bias. Furthermore, the outcome measures used may have some obvious limitations. For example, the PSC can provide recommendations for referral for further care and treatment, but cannot be used to enforce follow-up care (11). Fifthly, we acknowledge that 3-months follow-up is not long enough for the patient to reintegrate successfully and/or adapt in the community. More studies with 
longer-term follow-up are needed. Finally, assessing the impact on families/carers and the community, associated costs and the treatment responses were beyond the scope of this study.

In conclusion, this prospective study demonstrates effective and feasible implementation of the mPSC in routine clinical practice, for improved clinical care and appropriate referrals for targeted intervention. It added to the functional information provided by the other measures, including the ClinFIT. Most clinicians were satisfied with the PSC in assisting therapeutic decision-making. These findings add to the existing evidence for PSC to inform healthcare providers of factors affecting the everyday activity of $\mathrm{PwS}$, and to inform targeted use of limited healthcare resources. Furthermore, robust clinical trials with control groups are needed to assess the responsiveness of the PSC to longer-term change associated with improved patient outcomes.

\section{ACKNOWLEDGEMENTS}

The authors thank all the participants in this study and the interdisciplinary team at the rehabilitation ward of the Royal Park Campus, Royal Melbourne Hospital. The authors also thank Drs Pouya Hafezi and Julie Carlyle for discharge summary audits, and Ms Loren Oscari for project management and data entry. Disclosure: This study was supported by internal resources of the Rehabilitation Department, Royal Melbourne Hospital, Australia. No commercial party having a direct financial interest in the results of the research supporting this article has conferred, or will confer, a benefit upon the authors or upon any organization with which the authors are associated.

The authors have no conflicts of interest to declare.

\section{REFERENCES}

1. National Stroke Foundation. Clinical guidelines for stroke management. Melbourne, Australia: NSF; 2017.

2. Cadilhac DA, Carter R, Thrift AG, Dewey HM. Estimating the long-term costs of ischemic and hemorrhagic stroke for Australia: new evidence derived from the North East Melbourne Stroke Incidence Study (NEMESIS). Stroke 2009; 40: 915-921.

3. Abdul Aziz AF, Mohd Nordin NA, Ali MF, Abd Aziz NA, Sulong S, Aljunid SM. The integrated care pathway for post stroke patients (iCaPPS): a shared care approach between stakeholders in areas with limited access to specialist stroke care services. BMC Health Serv Res 2017; 17: 35.

4. Bahalla A, James M, Stanley K, Ralph S, Durante N, Mcmullen $E$, et al. Springboard for Progress: the Seventh SSNAP Annual Report. London (UK): Healthcare Quality Improvement Partnership (HQIP); 2021.

5. Andersen HE, Schultz-Larsen K, Kreiner S, Forchhammer $\mathrm{BH}$, Eriksen K, Brown A. Can readmission after stroke be prevented? Results of a randomized clinical study: a postdischarge follow-up service for stroke survivors. Stroke 2000; 31: 1038-1045.

6. Andersen HE, Eriksen K, Brown A, Schultz-Larsen K, Forchhammer $\mathrm{BH}$. Follow-up services for stroke survivors after hospital discharge - a randomized control study. Clin Rehabil 2002; 16: 593-603.
7. Markus H. Improving the outcome of stroke. BMJ 2007 ; 335: 359-360.

8. Martin BJ, Yip B, Hearty M, Marletta S, Hill R. Outcome, functional recovery and unmet needs following acute stroke. Experience of patient follow up at 6 to 9 months in a newly established stroke service. Scott Med J 2002; 47: 136-137.

9. Rodgers H, Dennis M, Cohen D, Rudd A. British Association of Stroke Physicians: benchmarking survey of stroke services. Age Ageing 2003; 32: 211-217.

10. Wissel J, Olver J, Sunnerhagen KS. Navigating the poststroke continuum of care. J Stroke Cerebrovasc Dis 2013; 22: $1-8$.

11. Iosa M, Lupo A, Morone G, Baricich A, Picelli A, Panza G, et al. Post Soft Care: Italian implementation of a post-stroke checklist software for primary care and identification of unmet needs in community-dwelling patients. Neurol Sci 2018; 39: 135-139.

12. McKevitt C, Fudge N, Redfern J, Sheldenkar A, Crichton S, Wolfe C. A stroke survivor needs survey 1-1-2010. London: The Stroke Association; 2010.

13. Murray J, Ashworth R, Forster A, Young J. Developing a primary care-based stroke service: a review of the qualitative literature. $\mathrm{Br}$ J Gen Pract 2003; 53: 137-142.

14. Philp I, Brainin M, Walker MF, Ward AB, Gillard $P$, Shields $A L$, et al. Development of a poststroke checklist to standardize follow-up care for stroke survivors. J Stroke Cerebrovasc Dis 2013; 22: e173-180.

15. Turner GM, Mullis R, Lim L, Kreit L, Mant J. Using a checklist to facilitate management of long-term care needs after stroke: insights from focus groups and a feasibility study. BMC Fam Pract 2019; 20: 2 .

16. Ward AB, Chen C, Norrving B, Gillard P, Walker MF, Blackburn $S$, et al. Evaluation of the Post Stroke Checklist: a pilot study in the United Kingdom and Singapore. Int J Stroke 2014; 9: 76-84.

17. Olver J, Yang S, Fedele B, Ni J, Frayne J, Shen G, et al. Post Stroke Outcome: global insight into persisting sequelae using the Post Stroke Checklist. J Stroke Cerebrovasc Dis 2021; 30: 105612.

18. Harrison PL, Hara PA, Pope JE, Young MC, Rula EY. The impact of postdischarge telephonic follow-up on hospital readmissions. Popul Health Manag 2011; 14: 27-32.

19. Amatya B, Elmalik A, Song K, Lee SY, Galea MP, Khan F. Implementation of the Clinical Functioning Information Tool (ClinFIT) in routine clinical practice: a feasibility Study J Rehabil Med 2022 (in press).

20. Knottnerus A, Tugwell P. STROBE - a checklist to Strengthen the Reporting of Observational Studies in Epidemiology. J Clin Epidemiol 2008; 61: 323.

21. Global Stroke Community Advisory Panel. Post-Stroke Checklist. 2012. [cited 201918 April; Available from: https://strokerecovery.ca/wp2019/wp-content/uploads/2020/07/HSF_PostStrokeChecklist_2pg_EN.pdf.

22. Ottenbacher KJ, Hsu Y, Granger CV, Fiedler RC. The reliability of the functional independence measure: a quantitative review. Arch Phys Med Rehabil 1996; 77: 1226-1232.

23. Cieza A, Oberhauser C, Bickenbach J, Chatterji S, Stucki G. Towards a minimal generic set of domains of functioning and health. BMC Public Health 2014; 14: 218.

24. Frontera $W$. The organizations of physical and rehabilitation medicine in the world: The International Society of Physical and Rehabilitation Medicine J Int Soc Phys Rehabil Med 2019: S130-133.

25. Prodinger B, Cieza A, Oberhauser C, Bickenbach J, Ustun TB, Chatterji S, et al. Toward the International Classification of Functioning, Disability and Health (ICF) Rehabilitation Set: a minimal generic set of domains for rehabilitation as a health strategy. Arch Phys Med Rehabil 2016; 97: 875-884.

26. Selb M, Gimigliano F, Prodinger B, Stucki G, Pestelli G, Iocco $M$, et al. Toward an International Classification of Functioning, Disability and Health clinical data collection 
p. 10 of 10 B. Amatya et al.

tool: the Italian experience of developing simple, intuitive descriptions of the Rehabilitation Set categories. Eur J Phys Rehabil Med 2017; 53: 290-298.

27. EuroQoL Group. EQ-5D-5L. [cited 201914 April]. Available from: https://euroqol.org/eq-5d-instruments/eq-5d-5Iabout/.

28. McColl MA, Davies D, Carlson P, Johnston J, Minnes P. The community integration measure: development and preliminary validation. Arch Phys Med Rehabil 2001; 82: 429-434.

29. Cohen J. Statistical power analysis for the behavioural sciences. 2nd edn. Hillsdale, NJ: Lawrence Erlbaum Associates, 1988.

30. Kinoshita S, Abo M, Okamoto T. Effectiveness of ICF-based multidisciplinary rehabilitation approach with serial assessment and discussion using the ICF rehabilitation set in a convalescent rehabilitation ward. Int J Rehabil Res 2020; 43: 255-260.

31. Kinoshita S, Abo M, Okamoto T, Kakuda W, Miyamura K, Kimura I. Responsiveness of the functioning and disability parts of the International Classification of Functioning, Disability, and Health core sets in postacute stroke patients. Int J Rehabil Res 2017; 40: 246-253.

32. Frontera W, Gimigliano F, Melvin J, Li J, Li L, Lains J, et al. ClinFIT: ISPRM's Universal Functioning Information Tool based on the WHO's ICF. J Int Soc Phys Rehabil Med 2019; 2: 19-21.

33. Mukaino M, Prodinger B, Yamada S, Senju Y, Izumi SI, Sonoda S, et al. Supporting the clinical use of the ICF in Japan - development of the Japanese version of the simple, intuitive descriptions for the ICF Generic-30 set, its operationalization through a rating reference guide, and interrater reliability study. BMC Health Serv Res 2020; 20: 66 .

34. Im HW, Kim WS, Kim S, Paik NJ. Prevalence of worsening problems using Post-Stroke Checklist and associations with quality of life in patients with stroke. J Stroke Cerebrovasc Dis 2020; 29: 105406.

35. Kjork EK, Carlsson G, Sunnerhagen KS, Lundgren-Nilsson A. Experiences using the poststroke checklist in Sweden with a focus on feasibility and relevance: a mixed-method design. BMJ Open 2019; 9: e028218.

36. Paolucci S, Smania N. Improving the quality of life of stroke survivors: what to do next? The Italian action for the implementation of a Poststroke Checklist. Eur J Phys Rehabil Med 2015; 51: 233-235.

37. Hotter B, Padberg I, Liebenau A, Knispel P, Heel S, Steube $D$, et al. Identifying unmet needs in long-term stroke care using in-depth assessment and the Post-Stroke Checklist - The Managing Aftercare for Stroke (MAS-I) study. Eur Stroke J 2018; 3: 237-245.

38. Ullberg T, Mansson K, Berhin I, Pessah-Rasmussen $H$. Comprehensive and structured 3-month stroke follow-up using the Post-stroke Checklist (The Struct-FU study): a feasibility and explorative study. J Stroke Cerebrovasc Dis 2021; 30: 105482 .

39. Delpont B, Blanc C, Osseby GV, Hervieu-Begue M, Giroud $M$, Bejot Y. Pain after stroke: a review. Rev Neurol (Paris) 2018; 174: 671-674.

40. Thomas LH, Coupe J, Cross LD, Tan AL, Watkins CL. Interventions for treating urinary incontinence after stroke in adults. Cochrane Database Syst Rev 2019; 2: CD004462. 